\title{
Non-Adiabatic Distortion In The Current Distribution Around A Moving Vortex
}

\author{
D.M. Gaitonde \\ Mehta Research Institute, \\ Chatnaag Road, Jhusi, \\ Allahabad 211019, INDIA.
}

\begin{abstract}
We study the phase distribution around a vortex in uniform motion. We consider both the cases of neutral and charged superfluids. The motion of the vortex causes the density of the system to fluctuate. This in turn produces a compensating current, thus ensuring current conservation locally. We explicitly calculate this current.
\end{abstract}


In recent years, there has been a great deal of interest (see Ref. 1 for a recent review) in the static and dynamic properties of vortices in superfluids and superconductors. A study of high temperature and other superconductors has led to the discovery of several new phenomena such as flux-lattice melting (2), quantum flux creep (3), anomalous sign change of the Hall co-efficient (4) and anomalous a.c. electromagnetic response(5). This has led to a renewed upsurge in efforts to understand the dynamics of moving vortices. Issues like the size and sign of the Magnus force (6) and the vortex inertial mass (7)have been intensely studied. Theoretical studies of these phenomena have been carried out, both within an effective Ginzburg-Landau theory (8) and microscopically (9) within the BCS theory.

However, most studies of vortex dynamics have tended to assume the vortex motion to be adiabatic i.e. the instantaneous distribution of the superconducting order parameter around the vortex is assumed to be identical with that of a vortex at rest. In this paper we address the question of non-adiabatic distortions in the phase distribution around a moving vortex. Our calculations are performed using a Ginzburg-Landau phase-only functional. We solve the equations of motion to second order in the vortex velocity $(u)$ and thus obtain corrections to the phase distribution.

The vortex motion induces fluctuations in the instantaneous density distribution around the vortex. Current conservation therefore causes a compensating current to flow, thus conserving the density locally. We explicitly calculate the induced current, to second order in $u$ for neutral as well as charged superfluids. The constant current contours are found to be distorted from their circular shape. However, there is no change in the energy, to second order in $u$, coming from the non-adiabatic distortions in the case of a neutral superfluid. For a charged superfluid the displacement current, associated with a time-dependent vector potential, contributes to a change in the energy. This contribution is suppressed by a factor of $u^{2} / c^{2}$ and is therefore negligibly small. These effects, thus have no consequences either for the Magnus force or for the vortex mass.

The superfluid is described by the action functional per unit length, $S^{\prime}=S / L=$ 
$\int d t \int d \vec{r}\left[\mathrm{~L}_{\theta}+\mathrm{L}_{\mathrm{em}}\right]$ where

$$
\mathrm{L}_{\theta}=\frac{\alpha_{1}}{2}\left(\dot{\theta}-\frac{2 e A_{0}}{\hbar}\right)^{2}-\frac{\alpha_{2}}{2}\left(\nabla \theta-\frac{2 e \vec{A}}{\hbar c}\right)^{2}+\gamma \dot{\theta}
$$

and

$$
\mathrm{L}_{\mathrm{em}}=\frac{\left(\nabla A_{0}+\frac{1}{c} \frac{\partial \vec{A}}{\partial t}\right)^{2}-(\nabla \times \vec{A})^{2}}{8 \pi}
$$

Here $\theta$ is the phase of the superconducting order parameter and $A_{0}$ and $\vec{A}$ are the scalar and vector potentials associated with the electromagnetic field. This functional has been derived microscopically (10) and the co-efficients $\alpha_{1}$ and $\alpha_{2}$ can be related to appropriate polarizabilities of the underlying fermionic system. The size and sign of the co-efficient $\gamma$, which determines the Magnus force, remains controversial. For purposes of this paper, we treat the Ginzburg-Landau coefficients as phenomenological parameters.

We will first consider the case of a neutral superfluid. Accordingly, we put $e=0$ in Eq. (1) and switch off the electromagnetic fields. We then have,

$$
L^{n s}=\frac{\alpha_{1}}{2} \dot{\theta}^{2}-\frac{\alpha_{2}}{2}(\nabla \theta)^{2}+\gamma \dot{\theta}
$$

The Euler-Lagrange equation of motion is easily found to be

$$
\alpha_{1} \ddot{\theta}=\alpha_{2} \nabla^{2} \theta
$$

Notice that the term linear in $\dot{\theta}$ corresponds to a topological phase and makes no contribution to the equation of motion.

For a uniformly moving vortex we have $\theta(\vec{r}, t)=\theta(\vec{r}-\vec{u} t)$. Substituting this in Eq. (3) we get

$$
\alpha_{1} \vec{u} \cdot \nabla(\vec{u} \cdot \nabla \theta)=\alpha_{2} \nabla \cdot(\nabla \theta)
$$

We seek a solution to Eq. (4) of the form

$$
\theta(\vec{r}, t)=\theta^{(0)}(\vec{r}-\vec{u} t)+\theta^{(1)}(\vec{r}-\vec{u} t)+\theta^{(2)}(\vec{r}-\vec{u} t)+\ldots
$$

where $\theta^{(m)}(\vec{r}-\vec{u} t)$ is of $m^{t h}$ order in $\vec{u}$. Here $\nabla \theta^{(0)}\left(\overrightarrow{r^{\prime}}\right)=\frac{\hat{\phi}^{\prime}}{r^{\prime}}$ (where we define the co-ordinate $\overrightarrow{r^{\prime}}=\vec{r}-\vec{u} t$ for convenience) is the phase distribution of a static vortex while the remaining 
terms are non-adiabatic phase distortions induced by the vortex motion. The quantization of the vorticity, which stems from the single-valuedness of the superconducting order parameter, ensures that for $m \neq 0 \theta^{(m)}\left(\overrightarrow{r^{\prime}}\right)$ is non-singular and $\nabla \theta^{(m)}$ is purely longitudinal.

Substituting Eq. (5) in Eq. (4) and equating terms of the same order in $u$, we arrive at the result

$$
\nabla^{\prime 2} \theta^{(1)}\left(\overrightarrow{r^{\prime}}\right)=0
$$

and

$$
\alpha_{1} \vec{u} \cdot \nabla^{\prime}\left(\vec{u} \cdot \nabla^{\prime} \theta^{(0)}\left(\overrightarrow{r^{\prime}}\right)\right)=\alpha_{2} \nabla^{\prime 2} \theta^{(2)}\left(\overrightarrow{r^{\prime}}\right)
$$

Since $\nabla \theta^{(1)}$ is constrained to be purely longitudinal, Eq. (6a) implies that it is zero. We therefore $\operatorname{set} \theta^{(1)}=0$.

Fourier transforming Eq. (6b) with respect to $\overrightarrow{r^{\prime}}$ we solve for $\theta_{\mathbf{q}}^{(2)}$ to get

$$
\theta_{\mathbf{q}}^{(2)}=\frac{-2 \pi \alpha_{1}}{\alpha_{2}} \vec{u} \cdot \vec{q} \frac{\vec{u} \cdot \hat{z} \times \vec{q}}{q^{4}}
$$

and find the corresponding current $\nabla^{\prime} \theta_{\mathbf{q}}^{(2)}$ to be

$$
\nabla^{\prime} \theta_{\mathbf{q}}^{(2)}=\frac{2 \pi \alpha_{1}}{\alpha_{2}} \vec{q}(\vec{u} \cdot \vec{q}) \frac{\vec{u} \cdot \hat{z} \times \vec{q}}{i q^{4}}
$$

It is easy to see from Eq. (2) that this current, which is purely longitudinal, doesn't contribute to the action to second order in $u$. This implies that both the Magnus force and the vortex mass are unaffected by the existence of this current. To get a better idea of the induced current distortion, we Fourier transform $\nabla^{\prime} \theta_{\mathbf{q}}$ (the details are outlined in the Appendix) to get

$$
\nabla^{\prime} \theta\left(\overrightarrow{r^{\prime}}\right)=\frac{\hat{\phi}^{\prime}}{r^{\prime}}\left[1-\frac{\alpha_{1} u^{2}}{2 \alpha_{2}} \cos 2 \phi^{\prime}\right]
$$

for the $\mathrm{x}$-axis chosen to be directed along $\vec{u}$. It is easily seen from Eq. (8) that the circular symmetry of a static vortex is destroyed by the extra current.

We now turn our attention to the charged case. The Euler-Lagrange equations of motion are now given by

$$
\alpha_{1} \frac{\partial}{\partial t}\left(\dot{\theta}-\frac{2 e}{\hbar} A_{0}\right)=\alpha_{2} \nabla \cdot\left(\nabla \theta-\frac{2 e}{\hbar c} \vec{A}\right)
$$




$$
\frac{\nabla \cdot\left(\nabla A_{0}+\frac{1}{c} \frac{\partial \vec{A}}{\partial t}\right)}{4 \pi}=-\frac{2 e \alpha_{1}}{\hbar}\left(\dot{\theta}-\frac{2 e}{\hbar} A_{0}\right)
$$

and

$$
\frac{\nabla \times(\nabla \times \vec{A})}{4 \pi}+\frac{1}{4 \pi c} \frac{\partial}{\partial t}\left(\nabla A_{0}+\frac{1}{c} \frac{\partial \vec{A}}{\partial t}\right)=\alpha_{2} \frac{2 e}{\hbar c}\left(\nabla \theta-\frac{2 e}{\hbar c} \vec{A}\right)
$$

We choose the gauge $\nabla \cdot \vec{A}=0$. Then, on Fourier transforming Eq. (9b) we find

$$
A_{0}(\vec{q})=\frac{\hbar}{2 e} \frac{\vec{u} \cdot \nabla^{\prime} \theta_{\vec{q}}}{q^{2} \lambda_{T F}^{2}+1}
$$

where the Thomas-Fermi screening length is given by $4 \pi \alpha_{1}\left(\frac{2 e}{\hbar}\right)^{2}=\lambda_{T F}^{-2}$. On Fourier transforming Eq. (9c) and making use of Eq. (10) we have

$$
\vec{A}(\vec{q})=\frac{(-\vec{u} \cdot \vec{q}) \vec{q} \hbar \vec{u} \cdot \nabla \theta_{\mathbf{q}}}{2 e c\left(q^{2} \lambda_{T F}^{2}+1\right)\left(q^{2}+\lambda_{L}^{-2}-(\vec{u} \cdot \vec{q} / c)^{2}\right)}+\frac{(\hbar c / 2 e) \nabla \theta_{\mathbf{q}}}{q^{2} \lambda_{L}^{2}+1-\left(\lambda_{L} \vec{u} \cdot \vec{q} / c\right)^{2}}
$$

where the penetration depth $\lambda_{L}$ is given by $4 \pi \alpha_{2}(2 e / \hbar c)^{2}=\lambda_{L}^{-2}$. In writing Eq. (11), we have made use of the relations $\vec{A}(\vec{r}, t)=\vec{A}(\vec{r}-\vec{u} t)$ and $A_{0}(\vec{r}, t)=A_{0}(\vec{r}-\vec{u} t)$. All Fourier transforms have been performed with respect to the co-ordinate $\overrightarrow{r^{\prime}}=\vec{r}-\vec{u} t$ as before.

We now substitute our result for $A_{0}$ (Eq.10) in Eq. (9a) and make use of our gauge condition to arrive at

$$
\alpha_{1} \vec{u} \cdot \vec{q} \vec{u} \cdot \nabla^{\prime} \theta_{\mathbf{q}} \frac{q^{2} \lambda_{T F}^{2}}{q^{2} \lambda_{T F}^{2}+1}=\alpha_{2} \vec{q} \cdot \nabla^{\prime} \theta_{\mathbf{q}}
$$

We put $\theta=\theta^{(0)}+\theta^{(1)}+\theta^{(2)}+\ldots$ as before. We once again find $\theta^{(1)}$ to be zero and get

$$
\theta_{\mathbf{q}}^{(2)}=-\frac{2 \pi \alpha_{1}}{\alpha_{2}} \vec{u} \cdot \vec{q} \frac{\vec{u} \cdot \hat{z} \times \vec{q}}{q^{4}} \frac{q^{2} \lambda_{T F}^{2}}{q^{2} \lambda_{T F}^{2}+1}
$$

Notice that as $e \rightarrow 0$ and $\lambda_{T F} \rightarrow \infty$, Eq.(13) recovers the earlier result of Eq. (7a) for a neutral superfluid.

Substituting Eq. (13) in Eq. (11) we find, to second order in $u$, the vector potential to be

$$
\vec{A}(\vec{q})=\frac{\hbar c}{2 e} \frac{2 \pi}{i q^{2}} \frac{\hat{z} \times \vec{q}}{q^{2} \lambda_{L}^{2}+1}\left[1+\frac{\lambda_{L}^{2}}{c^{2}} \frac{(\vec{u} \cdot \vec{q})^{2}}{q^{2} \lambda_{L}^{2}+1}\right]
$$

As expected, the vector potential is purely transverse as the longitudinal terms exactly cancel, consistent with our gauge condition. 
Making use of Eqs. (13) and (14) we find that the current density $\vec{j}\left(\overrightarrow{r^{\prime}}\right)=\left[\nabla^{\prime} \theta-\frac{2 e}{\hbar c} \vec{A}\right]$ is given by $\vec{j}=\vec{j}_{a}+\vec{j}_{b}+\vec{j}_{c}$ where

$$
\vec{j}_{a}(\vec{q})=\frac{2 \pi}{i q^{2}} \frac{\hat{z} \times \overrightarrow{q q^{2}} \lambda_{L}^{2}}{q^{2} \lambda_{L}^{2}+1}
$$

is the cuurent density of a vortex at rest,

$$
\vec{j}_{b}(\vec{q})=\frac{2 \pi \lambda_{L}^{2}}{i q^{2} c^{2}} \frac{\hat{z} \times \vec{q} q^{2} \lambda_{L}^{2}}{\left(q^{2} \lambda_{L}^{2}+1\right)^{2}}(\vec{u} \cdot \vec{q})^{2}
$$

corresponds to the "displacement current" of the moving flux and

$$
\vec{j}_{c}(\vec{q})=\frac{2 \pi \lambda_{L}^{2}}{i q^{2} c^{2}} \vec{q}(\vec{u} \cdot \vec{q}) \frac{\vec{u} \cdot \hat{z} \times \vec{q}}{q^{2} \lambda_{T F}^{2}+1}
$$

is the induced current necessary for local charge conservation.

We now proceed to evaluate $\vec{j}\left(\overrightarrow{r^{\prime}}\right)$. Fourier transforming $\vec{j}_{a}(\vec{q})$ we find (see Appendix for details)

$$
\vec{j}_{a}\left(\overrightarrow{r^{\prime}}\right)=\frac{\hat{\phi}^{\prime}}{\lambda_{L}} K_{1}\left(r^{\prime} / \lambda_{L}\right)
$$

which is the usual result for a vortex at rest. Here $K_{m}(x)$ are the modified Bessel functions. In a similar manner, we evaluate $\vec{j}_{b}\left(\overrightarrow{r^{\prime}}\right)$ and $\vec{j}_{c}\left(\overrightarrow{r^{\prime}}\right)$, details of which have been provided in the Appendix.

We find

$$
\overrightarrow{j_{b}}\left(\overrightarrow{r^{\prime}}\right)=\frac{u^{2}}{4 c^{2}}\left[\left(\left(2+\cos 2 \phi^{\prime}\right) I_{1}-\cos 2 \phi^{\prime} I_{2}\right) \hat{\phi}^{\prime}+\sin 2 \phi^{\prime}\left(I_{1}+I_{2}\right) \hat{r}^{\prime}\right]
$$

where

$$
I_{1}=\frac{1}{2 \lambda_{L}}\left[3 K_{1}\left(r^{\prime} / \lambda_{L}\right)+\frac{r^{\prime}}{\lambda_{L}} K_{1}^{\prime}\left(r^{\prime} / \lambda_{L}\right)\right]
$$

and

$$
I_{2}=\frac{r^{\prime}}{2 \lambda_{L}^{2}} K_{2}\left(r^{\prime} / \lambda_{L}\right)
$$

Similarly,

$$
\vec{j}_{c}\left(\overrightarrow{r^{\prime}}\right)=\frac{u^{2} \lambda_{L}^{2}}{4 c^{2}}\left[\left(I_{4}-I_{3}\right) \sin 2 \phi^{\prime} \hat{r}^{\prime}-\left(I_{3}+I_{4}\right) \cos 2 \phi^{\prime} \hat{\phi}^{\prime}\right]
$$

Here

$$
I_{3} \approx 0
$$


and

$$
I_{4} \approx \frac{8}{r^{\prime 3}}\left(1-J_{0}\left(r^{\prime} / \xi\right)\right)-\frac{8}{r^{\prime 2} \xi} J_{1}\left(r^{\prime} / \xi\right)+\frac{4}{r^{\prime} \xi^{2}} J_{1}^{\prime}\left(r^{\prime} / \xi\right)
$$

and $J_{m}(x)$ are Bessel functions. The smallness of $I_{3}$ and $I_{4}$ stems from the small screening length $\left(\lambda_{T F}\right)$ associated with $j_{c}$ (see Eq.(15c)). This length is much smaller than the coherence length $\xi$, which is the coarse-graining scale beyond which a description in terms of a phase-only action functional is applicable. Notice also that both $j_{b}$ and $j_{c}$ are strongly suppressed by factors of $u^{2} / c^{2}$ making their observation a difficult task.

We now consider the drawbacks of our calculation. As pointed out by Aitchison et. al. (10), at $T=0$, one has to include additional terms in the phase-only Lagrangian to ensure Galilean invariance. However, the solution of the corresponding non-linear problem is beyond the scope of our work. We have also ignored effects arising from the vortex core, whose proper inclusion would require a use of microscopic theory.

Finally, we summarise the main results of this paper. We have calculated the nonadiabatic phase distortion of a moving vortex.Vortex motion induces time-dependent density fluctuations, which in turn gives rise to additional currents necessary to ensure local charge conservation. For the neutral superfluid, the extra supercurrent is purely longitudinal with observable consequences. In the charged case there are two distinct contributions. One coming from the "displacement current" ( $j_{b}$ above) is purely transverse whereas the other ( $j_{c}$ above) is longitudinal. These currents are however strongly suppressed by factors of $u^{2} / c^{2}$ as well as efficient Coulomb screening in the case of $j_{c}$, making their observation a difficult task. 


\section{APPENDIX:}

In this appendix, we outline the derivation of the results stated above for the current $\vec{j}\left(\overrightarrow{r^{\prime}}\right)$. We will first take up the case of a neutral superfluid. Then we have

$$
\nabla^{\prime} \theta_{\mathbf{q}}=\frac{2 \pi \hat{z} \times \vec{q}}{i q^{2}}+\frac{2 \pi \alpha_{1}}{\alpha_{2}} \vec{q}(\vec{u} \cdot \vec{q}) \frac{\vec{u} \cdot \hat{z} \times \vec{q}}{i q^{4}}
$$

where the first term on the R.H.S. is the usual current of a vortex at rest and the second term is the distortion induced by the vortex motion. Thus, the current in real space is given by

$$
\nabla^{\prime} \theta\left(\overrightarrow{r^{\prime}}\right)=\int \frac{d \vec{q}}{(2 \pi)^{2}} \exp \left(i \vec{q} \cdot \overrightarrow{r^{\prime}}\right) \nabla^{\prime} \theta_{\mathbf{q}}
$$

The first term in Eq. (A1) is easily Fourier transformed to yield the standard result

$$
\nabla^{\prime} \theta\left(\overrightarrow{r^{\prime}}\right)_{a}=\frac{\hat{\phi}^{\prime}}{r^{\prime}}
$$

We find, on performing the angular integrals in Eq. (A2), the second term in the current to be

$$
\nabla^{\prime} \theta\left(\overrightarrow{r^{\prime}}\right)_{b}=\frac{-\alpha_{1} u^{2}}{4 \alpha_{2}} \int d q\left[\hat{r}^{\prime} \sin 2 \theta\left(J_{3}\left(q r^{\prime}\right)-J_{1}\left(q r^{\prime}\right)\right)+\hat{\phi}^{\prime} \cos 2 \theta\left(J_{3}\left(q r^{\prime}\right)+J_{1}\left(q r^{\prime}\right)\right)\right.
$$

where $\theta$ is the angle $\vec{u}$ makes with respect to $\overrightarrow{r^{\prime}}$. The integrals in Eq. (A4) can now be performed to yield

$$
\nabla^{\prime} \theta\left(\overrightarrow{r^{\prime}}\right)_{b}=\frac{-\alpha_{1} u^{2}}{2 \alpha_{2}} \cos 2 \theta \frac{\hat{\phi}^{\prime}}{r^{\prime}}
$$

On adding the results of Eqs. (A3) and (A5) and choosing $\vec{u}$ to lie along the $\mathrm{x}$-axis we finally arrive at Eq.(8) above.

We now consider the charged case. In this case, the current $\vec{j}(\vec{q})$ is the sum of three different contributions: $\vec{j}_{a}, \vec{j}_{b}$ and $\vec{j}_{c}$. The corresponding Fourier transforms $\vec{j}_{a}(\vec{q}), \vec{j}_{b}(\vec{q})$ and $\vec{j}_{c}(\vec{q})$ have been obtained earlier (Eq. (15)). We will evaluate each piece seperately.

$$
\vec{j}_{a}\left(\overrightarrow{r^{\prime}}\right)=\int \frac{d \vec{q}}{(2 \pi)^{2}} \exp \left(i \vec{q} \cdot \overrightarrow{r^{\prime}}\right) \frac{2 \pi}{i q^{2}} \frac{\hat{z} \times \vec{q} q^{2} \lambda_{L}^{2}}{q^{2} \lambda_{L}^{2}+1}
$$

is the usual current associated with a static vortex. On performing the angular integration we get

$$
\vec{j}_{a}\left(\vec{r}^{\prime}\right)=\hat{\phi}^{\prime} \int d q \frac{q^{2} J_{1}\left(q r^{\prime}\right)}{q^{2}+\lambda_{L}^{-2}}=\frac{\hat{\phi}^{\prime}}{\lambda_{L}} K_{1}\left(r^{\prime} / \lambda_{L}\right)
$$


We now consider $\overrightarrow{j_{b}}\left(\overrightarrow{r^{\prime}}\right)$.

$$
\vec{j}_{b}\left(\overrightarrow{r^{\prime}}\right)=\int \frac{d \vec{q}}{(2 \pi)^{2}} \exp \left(i \vec{q} \cdot \overrightarrow{r^{\prime}}\right) \frac{2 \pi \lambda_{L}^{2}}{i q^{2} c^{2}} \frac{\hat{z} \times \vec{q} q^{2} \lambda_{L}^{2}}{\left(q^{2} \lambda_{L}^{2}+1\right)^{2}}(\vec{u} \cdot \vec{q})^{2}
$$

On performing the angular integral we arrive at Eq. (17) above where

$$
I_{1}=\int d q \frac{q^{4} \lambda_{L}^{4} J_{1}(q r)}{\left(q^{2} \lambda_{L}^{2}+1\right)^{2}}
$$

and

$$
I_{2}=\int d q \frac{q^{4} \lambda_{L}^{4} J_{3}(q r)}{\left(q^{2} \lambda_{L}^{2}+1\right)^{2}}
$$

$I_{1}$ can be rewritten as

$$
I_{1}=\frac{-\lambda_{L}^{3}}{2} \frac{\partial}{\partial \lambda_{L}} \int d q \frac{q^{2} J_{1}\left(q r^{\prime}\right)}{q^{2} \lambda_{L}^{2}+1}=\frac{-\lambda_{L}^{3}}{2} \frac{\partial}{\partial \lambda_{L}}\left[\frac{K_{1}\left(r^{\prime} / \lambda_{L}\right)}{\lambda_{L}^{3}}\right]
$$

which reduces to the result of Eq. (18a). $I_{2}$ is directly evaluated to yield the result stated earlier in Eq. (18b).

Finally we consider $\vec{j}_{c}\left(\overrightarrow{r^{\prime}}\right)$.

$$
\vec{j}_{c}\left(\overrightarrow{r^{\prime}}\right)=\int \frac{d \vec{q}}{(2 \pi)^{2}} \exp \left(i \vec{q} \cdot \overrightarrow{r^{\prime}}\right) \frac{2 \pi \lambda_{L}^{2}}{i q^{2} c^{2}} \vec{q}(\vec{u} \cdot \vec{q}) \frac{\vec{u} \cdot \hat{z} \times \vec{q}}{q^{2} \lambda_{T F}^{2}+1}
$$

On performing the angular integration in Eq. (A11) we arrive at the result of Eq. where

$$
I_{3}=\int d q \frac{q^{2} J_{1}\left(q r^{\prime}\right)}{q^{2} \lambda_{T F}^{2}+1}
$$

and

$$
I_{4}=\int d q \frac{q^{2} J_{3}\left(q r^{\prime}\right)}{q^{2} \lambda_{T F}^{2}+1}
$$

$I_{3}$ is easily evaluated to yield

$$
I_{3}=\frac{1}{\lambda_{T F}^{3}} K_{1}\left(r^{\prime} / \lambda_{T F}\right)
$$

The Thomas-Fermi screening length $\lambda_{T F} \ll \xi$ where $\xi$ is the superconducting coherence length beyond which our coarse-grained picture based on a phase-only functional is valid. Thus $K_{1}\left(r^{\prime} / \lambda_{T F}\right) \approx \exp \left(-r^{\prime} / \lambda_{T F}\right) \rightarrow 0$ in this $(r>\xi)$ regime. 
To evaluate $I_{4}$ we make use of the relation $J_{m-1}(x)-J_{m+1}(x)=2 J_{m}^{\prime}(x)$ which is obeyed by Bessel functions. Using this relation, we find that $I_{4}$ can be rewritten as

$$
I_{4}=\int d q \frac{q^{2} J_{1}\left(q r^{\prime}\right)}{q^{2} \lambda_{T F}^{2}+1}-2 \frac{\partial}{\partial r^{\prime}} \int d q \frac{q\left[J_{0}\left(q r^{\prime}\right)-2 J_{1}^{\prime}\left(q r^{\prime}\right)\right]}{q^{2} \lambda_{T F}^{2}+1}
$$

This expression can be further simplified to yield

$$
I_{4}=\frac{1}{\lambda_{T F}^{3}} K_{1}\left(r^{\prime} / \lambda_{T F}\right)-2 \frac{\partial}{\partial r^{\prime}}\left[\frac{K_{0}\left(r^{\prime} / \lambda_{T F}\right)}{\lambda_{T F}^{2}}\right]+4 \frac{\partial^{2}}{\partial r^{\prime 2}} \int d q \frac{J_{1}\left(q r^{\prime}\right)}{q^{2} \lambda_{T F}^{2}+1}
$$

We ignore the first two terms on the right hand side of Eq. (A15) as they are exponentially small and in view of the small screening length, approximate the last integral by

$$
\int d q \frac{J_{1}\left(q r^{\prime}\right)}{\left(q^{2} \lambda_{T F}^{2}+1\right)} \approx \int_{0}^{\xi^{-1}} d q J_{1}\left(q r^{\prime}\right)=\frac{1}{r^{\prime}}\left[1-J_{0}\left(r^{\prime} / \xi\right)\right]
$$

Substituting these approximations in Eq. (A15) we finally arrive at the result of Eq. (20b). 


\section{References}

1. G. Blatter, M.V. Feigel'man, V.B. Geshkenbein, A.I. Larkin and V.M. Vinokur, Rev. Mod. Phys. 66, 1125 (1994).

2. A. Oral et. al., Phys. Rev. Lett. 80, 3610 (1998) and references therein.

3. A. F. Th. Hoekstra et. al., Phys. Rev. Lett. 80, 4293 (1998).

4. T. Nagaoka et. al., Phys. Rev. Lett. 80, 3594 (1998); S. Bhattacharya, M.J. Higgins and T.V. Ramakrishnan, Phys. Rev. Lett. 73, 1699 (1994).

5. S. Spielman et. al., Phys. Rev. Lett. 73, 1537 (1994).

6. D. J. Thouless, P. Ao and Q. Niu Phys. Rev. Lett. 76, 3758 (1996); G. E. Volovick JETP Lett. 62, 66 (1995).

7. D. M. Gaitonde and T. V. Ramakrishnan, Phys. Rev. B56, 11,951 (1997) and references therein.

8. J-M. Duan Phys. Rev. B48, 333 (1993).

9. A. van Otterlo, M. V. Feigelman, V. B. Geshkenbein and G. Blatter Phys. Rev. Lett. 75, 3736 (1995).

10. T. V. Ramakrishnan, Physica Scripta T 27, 24 (1989); I. J. R. Aitchison et. al., Phys. Rev. B 51, 6531 (1995). 